\title{
Enthalpies of Dilution of Polymer Solutions
}

\author{
Ichiro Fujihara, Katsutoshi Tamura, Sachio MuraKami, \\ and Ryoichi FuJISHIRo \\ Department of Chemistry, Faculty of Science, Osaka City University, \\ Sumiyoshi-ku, Osaka 558, Japan.
}

(Received April 19, 1978)

\begin{abstract}
Enthalpy of dilution for the polystyrene+cyclohexanone system was measured at $298 \mathrm{~K}$, using a dilution calorimeter, and the interaction energy parameter $X_{12}$, developed by Flory, was estimated. It was found from the results that, in comparison with the $X_{12}$ value of the polystyrene+cyclohexane system, that of the present system was more negative in the sequence: cyclohexanone $>$ methyl ethyl ketone, measured previously. The difference between hydrocarbon and carbonyl compounds was explained in terms of dipolar effects. Also, the dependence of $X_{12}$ on the molecular weight of polymer was briefly discussed in terms of "the correlation of molecular orientation" described by Patterson.

KEY WORDS Enthalpy of Dilution / Polystyrene / Methyl Ethyl Ketone / Cyclohexanone /
\end{abstract}

In a previous publication, ${ }^{1}$ we measured the enthalpy of dilution for the polystyrene (PS) + methyl ethyl ketone (MEK) system at 298K, using a newly constructed isothermal calorimeter and estimated the interaction energy parameter between the polymer and the solvent from the modified van Laar equation, and found it to depend on the molecular weight of polymer.

The present paper continues this investigation by reporting the enthalpy of dilution for the PS + cyclohexanone (CHK) system, in order to find if such behavior occurs in another system. The result is compared with the corresponding state theory developed by Flory.

\section{EXPERIMENTAL}

\section{Material}

Polystyrene samples used in this study, having molecular weights $(M)=2,200,4,000,9,000,37,000$, and 110,000, were obtained from Pressure Chemical Co. They have the narrow distribution of a molecular weight $\left(M_{w} / M_{n}\right.$ less than 1.06). Another PS sample of $M=22,000$, was supplied by Asahi Dow Chemical Co. The "Special Grade Reagent" CHK from Wako Pure Chemicals Co., Ltd. was distilled under reduced pressure. The purity of the solvent material was checked with an analytical gas-liquid chromatograph (Shimazu, Model GC-3BT) using a column containing PEG 6000 on Teflon. The result showed no significant impurity peaks by the highest sensitivity (full scale of $1 \mathrm{mv}$ ) of the detector.

\section{Calorimeter}

The enthalpy of dilution was measured at $298 \mathrm{~K}$ using a dilution isothermal calorimeter. ${ }^{1}$ The stirring effect due to the viscosity difference before and after dilution, brings a significant error into the enthalpy of dilution and hence the correction for this effect was made by the same procedure, as described previously. ${ }^{1}$ The systematic error inherent in the $\chi_{\mathrm{h}}$ value calculated from the experimental enthalpy of dilution at each dilution process is attributed mainly to (1) the estimation of the stirring effect described above, $\Delta \chi_{\mathrm{st}}$, (2) the determination of the partial molar volume of PS, $\Delta \chi_{\mathrm{rol}}$, and (3) the difference of the base line on the pen recorder monitoring the heat effect before and after dilution, $\Delta \chi_{\mathrm{b}}$. The total error $\Delta \chi=\Delta \chi_{\mathrm{st}}$ $\left.+\Delta \chi_{\text {rol }}+\Delta \chi_{\mathrm{b}}\right)$ is believed to be \pm 0.02 in maximum.

\section{RESULTS AND DISCUSSION}

The enthalpies of dilution of a PS solution, except for that of $M=110,000$, were measured in 
a concentration range from 0.2 to 0.1 in volume fraction of the polymer, by adding succesively a given amount of solvent to the PS solution of a known concentration. On the other hand, the enthalpy of dilution for PS solution of $M=110,000$, was measured from 0.07 to 0.04 in volume fraction. In order to determine the volume fraction of PS, the partial molar volume of PS, $\bar{v}_{2}{ }^{0}$ was measured pycnometrically at $298 \mathrm{~K}$. The result was 0.94 $\mathrm{cm}^{3} / \mathrm{g}$ for CHK. The reduced partial molar residual enthalpy $\chi_{h}$, was determined from the enthalpy of dilution measurement, using the following modified van Laar equation

$$
\chi_{\mathrm{h}}=\left(Q V_{1}^{0}\right) /\left(R T \Delta V \phi_{2} \phi_{2}{ }^{\prime}\right)
$$

where $V_{1}^{0}, R$, and $T$ are the molar volume of solvent, the gas constant, and the Kelvin temperature, respectively. $Q$ is the enthalpy change, obtained when the polymer solution is diluted from $\phi_{2}$ to $\phi_{2}{ }^{\prime}$ by adding the volume, $\Delta V$ of titre. Figure 1 shows the typical concentration dependence of $\chi_{\mathrm{h}}$ of the PS $(M=9,000)+$ CHK system.

It is well known that $\chi_{\mathrm{h}}$ depends on the concentration of polymer in solution. However, the experimental points are rather scattered, as shown in Figure 1. The attainment of a higher precision would be quite difficult in view of the very small heat effects to be measured. The $\chi_{\mathrm{h}}$ value was assumed to be independent of the concentration in this study and was averaged over the concentration range in which the enthalpy of dilution was measured.

In Figure 2, the values of $\chi_{\mathrm{h}}$ for the system

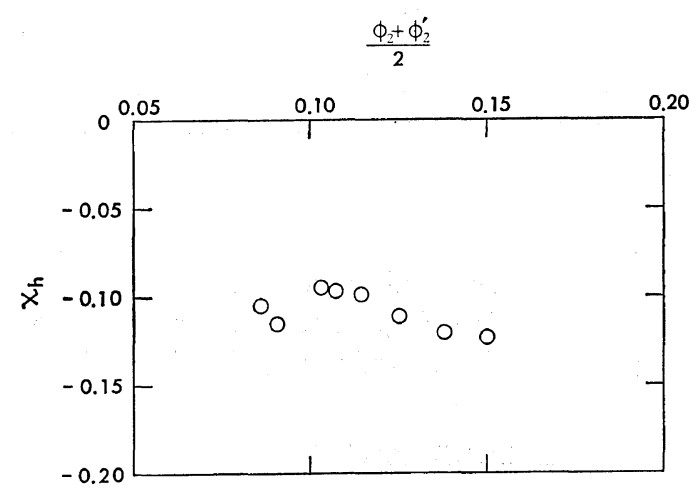

Figure 1. Concentration dependence of $\chi_{\mathrm{h}}$ of the polystyrene $(M=9,000)+$ cyclohexanone system.

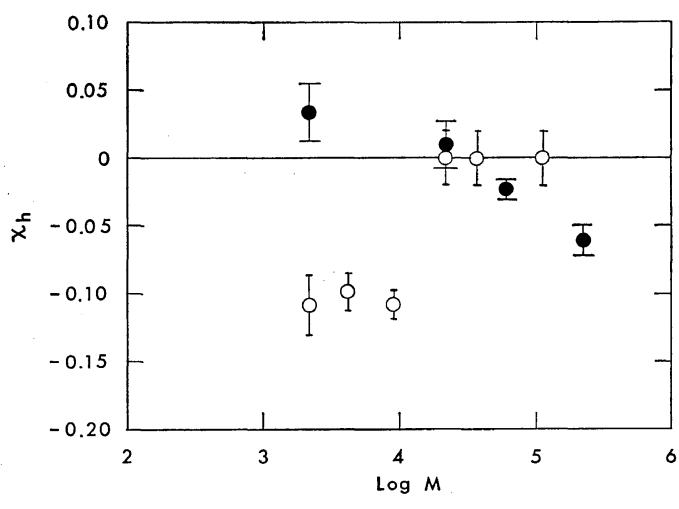

Figure 2. Dependence of $\chi_{\mathrm{h}}$ parameters of polystyrene solution on the molecular weight of polymer: polystyrene + methyl ethyl ketone; $\bigcirc$, polystyrene + cyclohexanone.

The vertical line represents the error contained in $\chi_{\mathrm{h}}$ value and that of the PS $(M=22,000,37,000$, and $110,000)+\mathrm{CHK}$ system represents the systematic error.

calculated by eq 1 are plotted against the logarithm of the molecular weight of PS, along with those of the PS + MEK system determined previously. ${ }^{1}$ The error contained in the $\chi_{\mathrm{h}}$ value, estimated from the assumption of the concentration independence at each point, is also indicated by the vertical line in Figure 2. As shown in this figure, the $\chi_{\mathrm{h}}$ values for both systems depend on the molecular weight of polymer, though they were averaged in the range of the measured concentration. This suggests a larger effect of the molecular weight than that of the concentration.

For the PS + MEK system, the $\chi_{\mathrm{h}}$ values change from positive to negative as the molecular weight of polymer increases. On the other hand, for the PS + CHK system, it is negative at the relatively low molecular weight region, while almost zero at a higher molecular weight region, and shows discontinuous behavior. Several systems showing anomalous behavior such as that of the latter system, have been recently found in the PS + organic solvents, containing oxygen or nitrogen atoms in the solvent molecule. ${ }^{2}$

In order to explain the molecular interaction in the polymer solution in this study, the experimental results were compared with the corresponding state theory developed by Flory. According to 
the Flory theory, the reduced partial molar residual enthalpy $\chi_{\mathrm{h}}$ is given by:

$$
\begin{gathered}
\chi_{\mathrm{h}}=\chi_{\mathrm{h}: 1}+\chi_{\mathrm{h}: 2} \phi_{2}+\chi_{\mathrm{h}: 3} \phi_{2}{ }^{2}+\cdots \\
\lim _{\phi_{2} \rightarrow 0} \chi_{\mathrm{h}}=\chi_{\mathrm{h}: 1}=\left(p_{1}{ }^{*} V_{1}{ }^{*} / \tilde{v}_{1} R T\right)\left[Y_{12}\left(1+\alpha_{1} T\right)\right. \\
\left.-(2 / 3) A^{2} \alpha_{1}{ }^{2} T^{2}\left(1+\alpha_{1} T\right)\right]
\end{gathered}
$$

where

$$
\begin{gathered}
A=\left(1-T_{1}{ }^{*} / T_{2}{ }^{*}\right)\left(p_{2}{ }^{*} / p_{1}{ }^{*}\right)-\left(s_{2} / s_{1}\right) X_{12} / p_{1}{ }^{*} \\
Y_{12}=X_{12} / p_{1}{ }^{*}\left(s_{1} / s_{2}\right)
\end{gathered}
$$

The notation in eq 2 to 5 closely follow those of Flory and reference should be made to the original paper for details of derivation. ${ }^{3}$

Table I. Equation-of-state data and characteristic parameters for methyl ethyl ketone, cyclohexanone, and polystyrene at $298 \mathrm{~K}$

\begin{tabular}{llll}
\hline \multicolumn{1}{r}{ Parameter } & MEK & \multicolumn{1}{c}{ CHK } & \multicolumn{1}{c}{ PS } \\
\hline$\alpha / 10^{-3} \mathrm{deg}^{-1}$ & 1.308 & 0.950 & 0.572 \\
$\tilde{v}$ & 1.3075 & 1.2373 & 1.1528 \\
$v / \mathrm{cm}^{3} \mathrm{~g}^{-1}$ & 1.2505 & 1.0616 & 0.9336 \\
$v^{*} / \mathrm{cm}^{3} \mathrm{~g}^{-1}$ & 0.9561 & 0.8580 & 0.8099 \\
$T^{*} / \mathrm{K}$ & 4557 & 5382 & 7420 \\
$V^{*} / \mathrm{cm}^{3} \mathrm{~mol}^{-1}$ & 68.94 & 84.21 & 84.35 \\
$p^{*} / \mathrm{J} \mathrm{cm} \mathrm{cm}^{-3}$ & 582 & 628 & 547 \\
$\tilde{T}$ & 0.06539 & 0.05537 & 0.04016 \\
\hline
\end{tabular}

The equation-of-state parameters required in the Flory calculation are shown in Table I. The values for PS and MEK were cited from the work of Höcker, et al. ${ }^{4}$ For CHK, they were calculated from the thermal expansion coefficient, $\alpha$, and the isothermal compressibility, $\beta$, in the literature. ${ }^{5,6}$ The ratio of the surface area of PS + solvent system, $s_{1} / s_{2}$ was calculated by assuming the solvent molecule as a sphere and the polymer as a cylinder. The results are as follows: 2.10 for PS + MEK, 1.97 for PS + CHK, respectively. Substituting the equation-of-state parameters in Table I and the $s_{1} / s_{2}$ value obtained above into eq 3 , the solutesolvent interaction energy parameter $X_{12}$, was determined to satisfy the experimental $\chi_{\mathrm{h}: 1}$ value in each system, and the results are shown in Figure 3.

$X_{12}$ is recognized as a measure expressing the weakness of the solute-solvent interaction relative to the solvent-solvent, and solute-solute interactions. In the non-polar mixture, it contains only the contribution from the dispersion force.

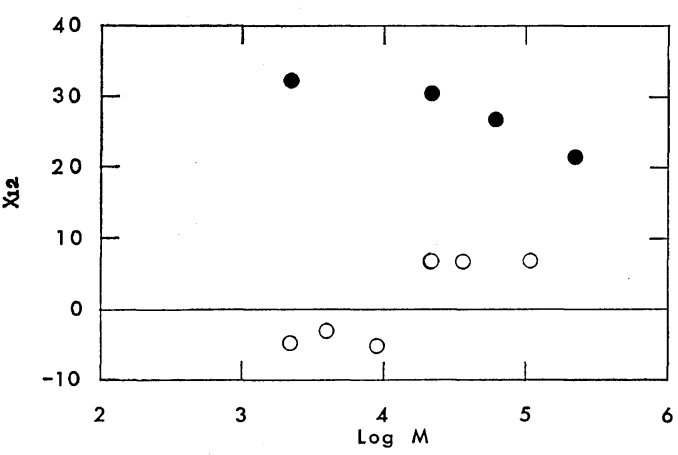

Figure 3. Dependence of $X_{12}$ parameters of polystyrene solution on the molecular weight of polymer: - polystyrene + methyl ethyl ketone; $\bigcirc$, polystyrene +cyclohexanone.

However, $X_{12}$ in this study should be considered to include the contribution not only from dispersion, but also from dipolar interaction. In order to evaluate the dipolar effect in this study, the $X_{12}$ value for the PS $+\mathrm{CHK}$ system was compared with that of the PS+cyclohexane system, in which cyclohexane molecule was regarded as a homologue of CHK molecule. Höcker, et al., estimated $X_{12}=$ $42 \mathrm{~J} / \mathrm{cm}^{3}$ for PS+cyclohexane system. ${ }^{4}$ On the other hand, the $X_{12}$ value for the PS + CHK system is obtained as negative. If the contribution of $X_{12}$ from the dispersion force is assumed to be nearly equal in two ketone systems, the different values of $X_{12}$ for the systems should be related to the difference of dipole moments of the solvent molecules. This assumption will be reasonable from the relatively small $X_{12}$ for the hydrocarbon mixtures. $^{3} \quad$ The dipole moments of the solvent molecules are as follows: 2.76D for MEK, 3.10D for CHK. The deviation of $X_{12}$ values from that of the PS + cyclohexane system lies in the sequence; MEK $<\mathrm{CHK}$, coinciding with the sequence of the dipole moments of the solvent molecules. In comparison with $X_{12}$ of the PS+cyclohexane system, the lowering of these values for the two systems in this investigation suggests that the PS molecule is energetically more stable in solution due to the dipole-induced dipole interaction between a carbonyl group of solvent molecule and phenyl group of PS molecule as described previously. ${ }^{7}$

Further inspection of Figure 2, reveals that the $X_{12}$ values change discontinuously with the mole- 
cular weight of polymer. According to the Flory theory, the $X_{12}$ parameter is defined in principle as independent of the molecular weight of polymer. Lam, et al., interpreted the effect of the size and shape for $X_{12}$ in terms of "the correlation of molecular orientation (CMO).", They associated $X_{12}$ with the force field difference between the components, and also with the difference in CMO, and assumed that the former effect appeared to be constant with the molecular weight of polymer, but that the latter one changed considerably in solutesolute contacts. A degree of orientational order should increase with the molecular weight of polymer and the polarity of solvent in the pure state. ${ }^{8}$ On the other hand, it will decrease or increase in the solution. While, in the present case, the order of molecular orientations increases by dipole-induced dipole interaction between solvent and PS molecule as described above, this will be destroyed largely with an increase in the molecular weight of the PS molecule, and the $X_{12}$ value changes toward the positive side with it. The PS molecule is well known to form a stacking structure between the benzene rings in a pure state. Such a structure will be destroyed by adding the solvent, and it is possible to form a new dipole-induced dipole interaction between solvent and PS molecule, and the degree of orientational order will increase. However, we have no information about how it depends on the molecular weight of polymer.

The equation of van Laar types has been used in the estimation of polymer-solvent interaction in solution from the enthalpy of dilution measurement. It is assumed in the derivation of the equation, that a polymer molecule is divided into several segments having the same size as that of a solvent molecule and the number of the effective nearestneighbour, and the segments mix with the solvent molecules randomly. The value of $\chi_{\mathrm{h}}$ in such model is originally independent of the concentration and the molecular weight of polymer. We obtained an expression of $\chi_{\mathrm{h}}$ dependent on the concentration, by using a parameter which reflects the size of a polymer molecule in solution. ${ }^{9}$ According to this expression, $\chi_{\mathrm{h}}$ at a very dilute concentration is dependent on $(M)^{-1 / 2}$ of polymer, while at a very high concentration, it is in agreement with that of the van Laar equation expressed by site fraction. The details of this treatment will be published some time in the near future.

\section{REFERENCES}

1. K. Tamura, S. Murakami, and R. Fujishiro, Polymer, 14, 237 (1973).

2. A. Kagemoto, private communication.

3. P. J. Flory, J. Am. Chem. Soc., 87, 1833 and 1838 (1965).

4. H. Höcker, G. J. Blake, and P. J. Flory, Trans. Faraday Soc., 67, 2251 (1971).

5. W. Schaaffs, "Molecular Acoustics" Vol. 5 in "Landolt-Börnstein New Series Group II." K.H. Hellwege and A.M. Hellwege, Ed., SpringerVerlag, Berlin, 1967.

6. J. A. Riddick and W. B. Bunger, "Techniques of Chemistry" Vol. 2 in "Organic Solvents," 3rd ed., Wiely-Interscience, New York, N. Y., 1970.

7. K. Tamura, S. Murakami, and R. Fujishiro, $J$. Chem. Thermodyn., 7, 1089 (1975).

8. V.T. Lam, P. Picker, D. Patterson, and P. Tancréde, J. Chem. Soc. Faraday, Trans. 2, 70, 1465 (1974).

9. I. Fujihara, K. Tamura, and S. Murakami, in preparation. 